Kragujevac Journal of Mathematics

Volume 38(1) (2014), Pages 163-170.

\title{
THE LAPLACIAN SPECTRUM OF CORONA OF TWO GRAPHS
}

\author{
QUN LIU ${ }^{1,2}$
}

\begin{abstract}
Let $G_{1}, G_{2}$ be two connected graphs. Denote the corona and the edge corona of $G_{1}, G_{2}$ by $G_{1} \circ G_{2}$ and $G=G_{1} \diamond G_{2}$, respectively. In this paper, we compute the Laplacian spectrum of the corona $G \circ H$ of two arbitrary graphs $G$ and $H$ and the edge corona of a connected regular graph $G_{1}$ and an arbitrary graph $G_{2}$.
\end{abstract}

\section{INTRODUCTION}

Throughout this paper, we consider only simple graphs. Let $G=(V(G), E(G))$ be a graph with vertex set $V(G)=\left\{v_{1}, v_{2}, \ldots, v_{n}\right\}$ and edge set $E(G)=\left\{e_{1}, e_{2}, \ldots, e_{m}\right\}$. The adjacency matrix of $G$ denoted by $A(G)$ is defined as $A(G)=\left(a_{i j}\right)$, where $\left(a_{i j}\right)=$ 1 if vertices $i$ and $j$ are adjacent in $G$ and 0 otherwise. The Laplacian matrix of $G$, denoted by $L(G)$ is defined as $D(G)-A(G)$, where $D(G)$ is the diagonal degree matrix of $G$. The Laplacian spectrum of $L(G)$ is defined as $L(G)=\left(\mu_{1}(G), \mu_{2}(G), \ldots, \mu_{n}(G)\right)$ where $0=\mu_{1}(G) \leq \mu_{2}(G) \leq \cdots \leq \mu_{n}(G)$ are the eigenvalues of $L(G)$. There is extensively literature available on works related to Laplacian spectrum of a graph. See $[4,5,6]$ and the references therein to know more.

The following two definitions come frow $[2,9]$. Let $G_{1}$ and $G_{2}$ be two graphs on disjoint sets of $n_{1}$ and $n_{2}$ vertices, $m_{1}$ and $m_{2}$ edges, respectively. The corona $G_{1} \circ G_{2}$ of $G_{1}$ and $G_{2}$ is defined as the graph obtained by taking one copy of $G_{1}$ and $n_{1}$ copies of $G_{2}$, and then joining the ith vertex of $G_{1}$ to every vertex in the ith copy of $G_{2}$. The edge corona $G_{1} \diamond G_{2}$ of $G_{1}$ and $G_{2}$ is defined as the graph obtained by taking one copy of $G_{1}$ and $m_{1}$ copies of $G_{2}$, and then joining two end-vertices of the ith edge of $G_{1}$ to every vertex in the i-th copy of $G_{2}$.

Key words and phrases. Laplacian matrix, Laplacian spectrum, Corona, edge corona, Kronecker product.

2010 Mathematics Subject Classification. Primary: 05C. Secondary: 05C12.

Received: May 3, 2013

Revised: October 6, 2013. 
Note that the corona $G_{1} \circ G_{2}$ has $n_{1}\left(n_{2}+1\right)$ vertices and $m_{1}+n_{1}\left(m_{2}+n_{2}\right)$ edges, and that the edge corona $G_{1} \diamond G_{2}$ has $n_{1}+m_{1} n_{2}$ vertices and $m_{1}+m_{1}\left(m_{2}+2 n_{2}\right)$ edges.

There have been some results on the corona and the edge corona of two graphs. Barik et al. [1] provided complete information about the adjacency spectrum of $G_{1} \circ G_{2}$ for a connected graph $G_{1}$ and a regular graph $G_{2}$, and complete information about the Laplacian spectrum of $G_{1} \circ G_{2}$ using the spectrum (and the Laplacian spectrum, respectively) of $G_{1}$ and $G_{2}$. In 2010, Hou and Shiu [7] considered the adjacency spectrum of $G_{1} \diamond G_{2}$ for a connected regular graph $G_{1}$ and a regular graph $G_{2}$ and the Laplacian spectrum of $G_{1} \diamond G_{2}$ for a connected regular graph $G_{1}$ and a graph $G_{2}$. Recently, McLeman and McNicholas [3], by introducing a new invariant called the coronal of a graph, also discussed the spectrum of $G_{1} \circ G_{2}$.

Motivated by these researches, we discuss the Laplacian spectrum of $G_{1} \circ G_{2}$ and $G_{1} \diamond G_{2}$. We also consider the spectrum of $G_{1} \diamond G_{2}$ when $G_{1}$ is regular. This paper is organized as follows. In Section 3, we introduce a new invariant, the L-coronal of a Laplacian matrix, and use it to compute the characteristic polynomial of the Laplacian matrix of $G_{1} \circ G_{2}$. Using this result, we give a complete description of the Laplacian eigenvalues of $G_{1} \circ G_{2}$ when $G_{1}$ is an arbitrary graph and $G_{2}$ is also an arbitrary graph. In Section 4, we give the characteristic polynomials of the Laplacian matrix of $G_{1} \diamond G_{2}$ for a regular graph $G_{1}$ and any graph $G_{2}$. Using these results, we give a complete description of Laplacian eigenvalues of $G_{1} \diamond G_{2}$ when $G_{1}$ is an $r_{1}$-regular graph and $G_{2}$ is an arbitrary graph.

\section{Preliminaries}

The Kronecker product of matrices $A=\left(a_{i j}\right)$ and $B$, denoted by $A \otimes B$, is defined to be the partition matrix $\left(a_{i j} B\right)$. See [8]. In cases where each multiplication makes sense, we have $M_{1} M_{2} \otimes M_{3} M_{4}=\left(M_{1} \otimes M_{3}\right)\left(M_{2} \otimes M_{4}\right)$.

This implies that for nonsingular matrix $M$ and $N,(M \otimes N)^{-1}=M^{-1} \otimes N^{-1}$, Recall also that for square matrices $M$ and $N$ of order $k$ and $s$, respectively. $\operatorname{det}(M \otimes N)=$ $(\operatorname{det} M)^{k}(\operatorname{det} N)^{s}$

If $M_{4}$ is invertible, then

$$
\operatorname{det}\left(\begin{array}{ll}
M_{1} & M_{2} \\
M_{3} & M_{4}
\end{array}\right)=\operatorname{det}\left(M_{4}\right) \operatorname{det}\left(M_{1}-M_{2} M_{4}^{-1} M_{3}\right) .
$$

Let $j_{n}$ be the column vector of size $n$ with all entries equal to one, $0_{n}$ a column zero vector of size $n$, and $I_{n}$ the identity matrix of order $n$. Let $e_{i}$ be the $i$-th unit column vector of size $n_{1}$ for $i=1,2, \cdots, n_{1}$. For a vertex $u$ of a graph $G$, let $d_{G}(u)$ be the degree of vertex $u$ in $G$. For vertices $u$ and $v$ in a graph, $u \sim v$ means that $v$ is adjacent to $u$.

For graphs $G_{1}$ with $n_{1}$ vertices and $G_{2}$ with $n_{2}$ vertices we have

$$
L\left(G_{1}\right)=\left(\mu_{1}, \mu_{2}, \cdots, \mu_{n_{1}}\right), L\left(G_{2}\right)=\left(\delta_{1}, \delta_{2}, \cdots, \delta_{n_{2}}\right) .
$$




\section{The LAPlacian SPECTRUM of CORONA}

In [3], McLeman and McNicholas introduced a new invariant, the coronal of a graph $G$ of order $n$. It is defined to be the sum of the entries of the matrix $\left(\lambda I_{n}-A\right)^{-1}$, where $I_{n}$ and $A$ are the identity matrix of order $n$ and the adjacency matrix of $G$, respectively. Now we shall generalize this concept to Laplacian matrix of graph $G$.

For a graph $G$ on $n$ vertices, the Laplacian characteristic polynomial of $G$ is $f_{G}(\lambda)=$ $\operatorname{det}\left(\lambda I_{n}-L(G)\right)$.

Definition 3.1. Let $G$ be a graph on $n$ vertices, with the Laplacian matrix $L(G)$. Note that, viewed as a matrix over the field of rational functions $C(\lambda)$, the characteristic matrix $\left((\lambda-1) I_{n}-L(G)\right)$ is invertible since its determinant $\operatorname{det}\left((\lambda-1) I_{n}-L(G)\right)=$ $f_{G}(\lambda-1) \neq 0$, The $L$-coronal $\chi_{H}(\lambda) \in C(\lambda)$ of $G$ is defined to be the sum of the entries of the matrix. Note this can be calculated as $\chi_{G}(\lambda)=j_{n}^{T}\left((\lambda-1) I_{n}-L(G)\right)^{-1} j_{n}$.

Our main theorem is that, beyond the spectra of $G$ and $H$, only the coronal of $H$ is needed to compute the spectrum of $G=G \circ H$.

Let $G_{1}$ be a graph with $V\left(G_{1}\right)=\left\{1,2, \cdots, n_{1}\right\}$ and $G_{2}$ be a graph with $n_{2}$ vertices. Let $G=G_{1} \circ G_{2}$. Then

$$
\begin{aligned}
A(G) & =\left(\begin{array}{cc}
A\left(G_{1}\right) & j_{n_{2}}^{T} \otimes I_{n_{1}} \\
\left(j_{n_{2}}^{T} \otimes I_{n_{1}}\right)^{T} & A\left(G_{2}\right) \otimes I_{n_{1}}
\end{array}\right) \\
L(G) & =\left(\begin{array}{cc}
L\left(G_{1}\right)+n_{2} I_{n_{1}} & -j_{n_{2}}^{T} \otimes I_{n_{1}} \\
-\left(j_{n_{2}}^{T} \otimes I_{n_{1}}\right)^{T} & \left(I_{n_{2}}+L\left(G_{2}\right) \otimes I_{n_{1}}\right.
\end{array}\right)
\end{aligned}
$$

Theorem 3.1. Let $G_{1}$ be a graph with $n_{1}$ vertices, $G_{2}$ be a graph with $n_{2}$ vertices. Let $\chi_{G_{2}}(\lambda)$ be the L-coronal of $G_{2}$. Then

$$
f_{G_{1} \circ G_{2}}(\lambda)=\left(f_{G_{2}}(\lambda-1)\right)^{n_{1}} f_{G_{1}}\left(\lambda-n_{2}-\chi_{G_{2}}(\lambda)\right) .
$$

In particular, the Laplacian characteristic polynomial of $G_{1} \circ G_{2}$ is completely determined by the Laplacian characteristic polynomials $f_{G_{1}}(\lambda)$ and $f_{G_{2}}(\lambda)$ and the $L$ coronal of $\chi_{G_{2}}(\lambda)$.

Proof. It is easily seen that

$$
\begin{aligned}
f_{G_{1} \circ G_{2}}(\lambda)= & \operatorname{det}\left(\lambda I_{n_{1}\left(n_{2}+1\right)}-L\left(G_{1} \circ G_{2}\right)\right) \\
= & \operatorname{det}\left(\begin{array}{cc}
\lambda I_{n_{1}}-L\left(G_{1}\right)-n_{2} I_{n_{1}} & j_{n_{2}}^{T} \otimes I_{n_{1}} \\
\left(j_{n_{2}}^{T} \otimes I_{n_{1}}\right)^{T} & \lambda I_{n_{1} n_{2}}-\left(I_{n_{2}}+L\left(G_{2}\right)\right) \otimes I_{n_{1}}
\end{array}\right) \\
= & \operatorname{det}\left(\begin{array}{cc}
\lambda I_{n_{1}}-L\left(G_{1}\right)-n_{2} I_{n_{1}} & j_{n_{2}}^{T} \otimes I_{n_{1}} \\
\left(j_{n_{2}}^{T} \otimes I_{n_{1}}\right)^{T} & \left(\lambda I_{n_{2}}-\left(I_{n_{2}}+L\left(G_{2}\right)\right) \otimes I_{n_{1}}\right.
\end{array}\right) \\
= & \operatorname{det}\left(\left(\lambda I_{n_{2}}-\left(I_{n_{2}}+L\left(G_{2}\right)\right)\right) \otimes I_{n_{1}}\right) \times \operatorname{det}\left(\lambda I_{n_{1}}-L\left(G_{1}\right)-n_{2} I_{n_{1}}\right. \\
& -\left(j_{n_{2}}^{T} \otimes I_{n_{1}}\right)\left(\left(\lambda I_{n_{2}}-\left(I_{n_{2}}+L\left(G_{2}\right)\right)\right) \otimes I_{n_{1}}\right)^{-1}\left(j_{n_{2}}^{T} \otimes I_{n_{1}}\right)^{T}
\end{aligned}
$$




$$
\begin{aligned}
= & \operatorname{det}\left((\lambda-1) I_{n_{2}}-L\left(G_{2}\right)\right)^{n_{1}} \times \operatorname{det}\left(\lambda I_{n_{1}}-L\left(G_{1}\right)-n_{2} I_{n_{1}}\right. \\
& \left.\left.-\left(j_{n_{2}}^{T}\left(\lambda I_{n_{2}}-I_{n_{2}}-L\left(G_{2}\right)\right)^{-1}\right) j_{n_{2}}\right) \otimes I_{n_{1}}\right) \\
= & \operatorname{det}\left((\lambda-1) I_{n_{2}}-L\left(G_{2}\right)\right)^{n_{1}} \operatorname{det}\left(\left(\lambda-\chi_{G_{2}}(\lambda)-n_{2}\right) I_{n_{1}}-L\left(G_{1}\right)\right) \\
= & \left(f_{G_{2}}(\lambda-1)\right)^{n_{1}} f_{G_{1}}\left(\lambda-n_{2}-\chi_{G_{2}}(\lambda)\right),
\end{aligned}
$$

as desired.

The following Theorem 3.2, first addressed in [1], is an immediate consequence of Theorem 3.1. We remark that here our method is straight-forward and different from that of Theorem 2.4 .

Theorem 3.2. Let $G_{1}$ be any graph with $n_{1}$ vertices, $m_{1}$ edges and $G_{2}$ be any graph with $n_{2}$ vertices, $m_{2}$ edges. Suppose that $L\left(G_{1}\right)=\left(\mu_{1}, \mu_{2}, \ldots, \mu_{n_{1}}\right)$ and $L\left(G_{2}\right)=$ $\left(\delta_{1}, \delta_{2}, \ldots, \delta_{n_{2}}\right)$. Then the Laplacian spectrum of $G_{1} \circ G_{2}$ is given by

(i) The eigenvalue $\delta_{j}+1$ with multiplicity $n_{1}$ for every eigenvalue $\delta_{j}\left(j=2, \ldots, n_{2}\right)$ of $L\left(G_{2}\right)$,

(ii) Two multiplicity-one eigenvalues $\frac{\left(\mu_{i}+n_{2}+1\right) \pm \sqrt{\left(n_{2}+1+\mu_{i}\right)^{2}-4 \mu_{i}}}{2}$ for each eigenvalue $\mu_{i}\left(i=1,2, \ldots, n_{1}\right)$ of $L\left(G_{1}\right)$.

Proof. Since the sum of all entries on every row of Laplacian matrix is zero, we have $L\left(G_{2}\right) j_{n_{2}}=0 j_{n_{2}}$, and then $\left((\lambda-1) I_{n_{2}}-L\left(G_{2}\right)\right) j_{n_{2}}=(\lambda-1) j_{n_{2}}$. Thus

$$
\chi_{G_{2}}(\lambda)=j_{n_{2}}^{T}\left((\lambda-1) I_{n_{2}}-L\left(G_{2}\right)\right)^{-1} j_{n_{2}}=\frac{j_{n_{2}}^{T} j_{n_{2}}}{\lambda-1}=\frac{n_{2}}{\lambda-1}
$$

The only pole of $\chi_{G_{2}}(\lambda)$ is $\lambda=1$. By Theorem 3.1, $\delta_{j}+1$ is an eigenvalue of $L\left(G_{1} \circ G_{2}\right)$ with multiplicity of $n_{1}$ for $j=2, \ldots, n_{2}$. The remaining $2 n_{1}$ eigenvalues are obtained by solving $\lambda-n_{2}-\frac{n_{2}}{\lambda-1}=\mu_{i}$, the theorem is proved.

Now, we give an alternative proof of the above theorem by an idea from [10].

Theorem 3.3. Let $G_{1}$ be a graph with $n_{1}$ vertices and $G_{2}$ be a graph with $n_{2}$ vertices, and their Laplacian spectrum are as in (2.1). Let

$$
\lambda_{i}, \overline{\lambda_{i}}=\frac{\left(\mu_{i}+n_{2}+1\right) \pm \sqrt{\left(n_{2}+1+\mu_{i}\right)^{2}-4 \mu_{i}}}{2}
$$

for $i=1,2, \ldots, n_{1}$. Then $L(G)$ is

$$
\left(\begin{array}{ccccccccc}
\delta_{1}+1, & \delta_{2}+1, & \ldots & \delta_{n_{2}-1}+1, & \lambda_{1}, & \overline{\lambda_{1}}, & \ldots & \lambda_{n_{1}}, & \overline{\lambda_{n_{1}}} \\
n_{1}, & n_{1}, & \ldots & n_{1}, & 1, & 1, & \ldots & 1, & 1
\end{array}\right),
$$

where entries in the first row are the eigenvalues with the multiplicities written below.

Proof. Let $Z_{1}, Z_{2}, \ldots, Z_{n_{2}}$ be the orthogonal of $L\left(G_{2}\right)$ corresponding to the eigenvalue $0=\delta_{1}, \delta_{2}, \ldots, \delta_{n_{2}}$, respectively. Then for $i=1,2, \ldots, n_{1}$ and for $k=2, \ldots, n_{2}$, we 
have

$$
\begin{aligned}
& L(G)\left(\begin{array}{c}
0_{n_{1}} \\
Z_{k} \otimes e_{i}
\end{array}\right)=\left(\begin{array}{c}
0_{n_{1}} \\
\left.\left(I_{n_{2}}+L\left(G_{2}\right)\right) \otimes I_{n_{1}}\right)\left(Z_{k} \otimes e_{i}\right)
\end{array}\right) \\
& =\left(\begin{array}{c}
0_{n_{1}} \\
\left(I_{n_{2}}+L\left(G_{2}\right)\right) Z_{k} \otimes I_{n_{1}} e_{i}
\end{array}\right) \\
& =\left(\delta_{k}+1\right)\left(\begin{array}{c}
0_{n_{1}} \\
Z_{k} \otimes e_{i}
\end{array}\right)
\end{aligned}
$$

and thus we obtain $n_{1}\left(n_{2}-1\right)$ eigenvalues and corresponding eigenvectors of $L(G)$.

Let $X_{1}, X_{2}, \ldots, X_{n_{1}}$ be the orthogonal eigenvectors of $L\left(G_{1}\right)$ corresponding to the eigenvalues $\mu_{1}, \mu_{2}, \ldots, \mu_{n_{1}}$, respectively. Let $F_{i}=\left(X_{i}^{T}, \frac{X_{i}^{T}}{1-\lambda_{i}}, \ldots, \frac{X_{i}^{T}}{1-\lambda_{i}}\right)$ for $i=$ $1,2, \ldots, n_{1}$. For any vertex $u$ in the $s$-th copy of $G_{2}, F_{i}(u)=\frac{X_{i}(s)}{1-\lambda_{i}}$, and thus

$$
d_{G}(u) F_{i}(u)-\sum_{v \sim u} F_{i}(v)=\left(d_{G_{2}}(u)+1\right) F_{i}(u)-X_{i}(s)-d_{G_{2}}(u) \frac{X_{i}(s)}{1-\lambda_{i}}=\lambda_{i} F_{i}(u) .
$$

For any vertex $u$ in $G_{1}$, we have $d_{G_{1}}(u) X_{i}(u)-\sum_{v \sim u, v \in V\left(G_{1}\right)} X_{i}(v)=\mu_{i} X_{i}(u)$, thus

$$
\begin{aligned}
d_{G}(u) F_{i}(u)-\sum_{v \sim u} F_{i}(v) & =\left(d_{G_{1}}(u)+n_{2}\right) X_{i}(u)-\sum_{v \sim u, v \in V\left(G_{1}\right)} X_{i}(v)-\frac{n_{2}}{1-\lambda_{i}} X_{i}(v) \\
& =\left(d_{G_{1}}(u)+n_{2}\right) F_{i}(u)-\sum_{v \sim u, v \in V\left(G_{1}\right)} F_{i}(v)-\sum_{v \sim u, v \notin V\left(G_{1}\right)} F_{i}(v) \\
& =\left(d_{G_{1}}(u)+n_{2}-\frac{n_{2}}{1-\lambda_{i}}\right) X_{i}(u)+\left(\mu_{i}-d_{G_{1}}(u)\right) X_{i}(u) \\
& =\lambda_{i} X_{i}(u)=\lambda_{i} F_{i}(u)
\end{aligned}
$$

It follows that $d_{G_{1}}(u) F_{i}(u)-\sum_{v \sim u} F_{i}(v)=\lambda_{i} F_{i}(u)$ for every vertex $u$ in $G$. Similarly, if $\overline{F_{i}}=\left(X_{i}^{T}, \frac{X_{i}^{T}}{1-\overline{\lambda_{i}}}, \ldots, \frac{X_{i}^{T}}{1-\overline{\lambda_{i}}}\right)$, then $d_{G}(u) \overline{F_{i}(u)}-\sum_{v \sim u} F_{i}(u)=\mu_{i} F_{i}(u)$ for every vertex $u$ in $G$. Therefore we obtain $2 n_{1}$ eigenvalues $\lambda_{i}, \overline{\lambda_{i}}$ and corresponding eigenvectors $F_{i}$ and $\overline{F_{i}}$ of $L(G)$ for $i=1,2, \ldots, n_{1}$.

Now we have obtained $n_{1}\left(n_{2}+1\right)$ eigenvalues and corresponding eigenvectors of $L(G)$ and it is easy to see that these eigenvectors of $L(G)$ are linearly independent. Hence, the proof is completed.

\section{The Spectrum And Laplacian spectrum of $G_{1} \diamond G_{2}$}

Let $G_{1}$ be a $r_{1}-$ regular graph with $n_{1}$ vertices, $m_{1}$ edges and $G_{2}$ be any graph with $n_{2}$ vertices, $m_{2}$ edges. Also let $L\left(G_{1}\right)$ and $L\left(G_{2}\right)$ be the Laplacian matrices of the graphs $G_{1}$ and $G_{2}$, respectively. Then the Laplacian matrix of $G=G_{1} \diamond G_{2}$ is

$$
L(G)=\left(\begin{array}{cc}
L\left(G_{1}\right)+r_{1} n_{2} I_{n_{1}} & -R\left(G_{1}\right) \otimes j_{n_{2}} \\
-\left(R\left(G_{1}\right) \otimes j_{n_{2}}\right)^{T} & I_{n_{1}} \otimes\left(2 I_{n_{2}}+L\left(G_{2}\right)\right.
\end{array}\right)
$$


where $R\left(G_{1}\right)=\left(r_{i j}\right)$ is the vertex-edge incident matrix with entry $r_{i j}=1$ if the vertex $i$ is incident the edge $e_{j}$ and 0 otherwise.

Theorem 4.1. Let $G_{1}$ be an $r_{1}$ - regular graph with $n_{1}$ vertices, $m_{1}$ edges and $G_{2}$ be any graph with $n_{2}$ vertices, $m_{2}$ edges. Also let $\chi_{G_{2}}(\lambda)$ be the $L$-coronal of $G_{2}$. Then

$$
f_{G}(\lambda)=\left[f_{G_{2}}(\lambda-2)\right]^{m_{1}} f_{G_{1}}\left(\frac{\lambda-r_{1} n_{2}-2 r_{1} \chi_{G_{2}}(\lambda-1)}{1-\chi_{G_{2}}(\lambda-1)}\right)\left[1-\chi_{G_{2}}(\lambda-1)\right]^{n_{1}} .
$$

In particular, the Laplacian characteristic polynomial of $G_{1} \diamond G_{2}$ is completely determined by the characteristic polynomials $f_{G_{1}}(\lambda)$ and $f_{G_{2}}(\lambda)$ and the L-coronal of $\chi_{G_{2}}(\lambda)$.

Proof. Note that, viewed as a matrix over the field of rational functions $C(\lambda)$, the following equalities make sense. If $\lambda$ is not a pole of $\chi_{G_{2}}(\lambda-1)$, then

$$
\begin{aligned}
f_{G_{1} \diamond G_{2}}(\lambda) & =\operatorname{det}\left(\lambda I_{n_{1}+m_{1} n_{2}}-L\left(G_{1} \diamond G_{2}\right)\right) \\
& =\operatorname{det}\left(\begin{array}{cc}
\lambda I_{n_{1}}-\left(L\left(G_{1}\right)+r_{1} n_{2} I_{n_{1}}\right) & R\left(G_{1}\right) \otimes j_{n_{2}} \\
\left(R\left(G_{1}\right) \otimes j_{n_{2}}\right)^{T} & \lambda I_{m_{1} n_{2}}-\left(I_{m_{1}} \otimes\left(2 I_{n_{2}}+L\left(G_{2}\right)\right)\right.
\end{array}\right) \\
& =\operatorname{det}\left(\begin{array}{cc}
\left(\lambda-r_{1} n_{2}\right) I_{n_{1}}-L\left(G_{1}\right) & R\left(G_{1}\right) \otimes j_{n_{2}} \\
\left(R\left(G_{1}\right) \otimes j_{n_{2}}\right)^{T} & I_{m_{1}} \otimes\left((\lambda-2) I_{n_{2}}-L\left(G_{2}\right)\right)
\end{array}\right) \\
& =\operatorname{det}\left(I_{m_{1}} \otimes\left((\lambda-2) I_{n_{2}}-L\left(G_{2}\right)\right) \times \operatorname{det} B,\right.
\end{aligned}
$$

where $B=\left(\lambda I_{n_{1}+m_{1} n_{2}}-L\left(G_{1}\right) /\left(I_{m_{1}} \otimes\left((\lambda-2) I_{n_{2}}-L\left(G_{2}\right)\right)\right.\right.$ is the Schur complement with respect to $I_{m_{1}} \otimes\left((\lambda-2) I_{n_{2}}-L\left(G_{2}\right)\right)$. Using many elementary results of Kronecker product of matrices, one has $\operatorname{det}\left(I_{m_{1}} \otimes\left((\lambda-2) I_{n_{2}}-L\left(G_{2}\right)\right)=\left((\lambda-2) I_{n_{2}}-L\left(G_{2}\right)\right)^{m_{1}}\right.$ and

$$
\begin{aligned}
\operatorname{det} B= & \operatorname{det}\left[\left(\lambda I_{n_{1}+m_{1} n_{2}}-L\left(G_{1}\right) /\left(I_{m_{1}} \otimes\left((\lambda-2) I_{n_{2}}-L\left(G_{2}\right)\right)\right]\right.\right. \\
= & \operatorname{det}\left\{\left(\lambda-r_{1} n_{2}\right) I_{n_{1}}-L\left(G_{1}\right)-\left(R\left(G_{1}\right) \otimes j_{n_{2}}\right)\right. \\
& {\left[\left(I_{m_{1}}^{-1} \otimes\left((\lambda-2) I_{n_{2}}-L\left(G_{2}\right)\right]^{-1}\left(R\left(G_{1}\right) \otimes j_{n_{2}}\right)^{T}\right\}\right.} \\
= & \operatorname{det}\left\{\left(\lambda-r_{1} n_{2}\right) I_{n_{1}}-L\left(G_{1}\right)-\left(R\left(G_{1}\right) I_{m_{1}} R\left(G_{1}\right)^{T}\right)\right. \\
& \left.\otimes\left(j_{n_{2}}\left((\lambda-2) I_{n_{2}}-L\left(G_{2}\right)\right)^{-1} j_{n_{2}}^{T}\right)\right\} \\
= & \operatorname{det}\left\{\left(\lambda-r_{1} n_{2}\right) I_{n_{1}}-L\left(G_{1}\right)-\left(2 r_{1} I_{n_{1}}-L\left(G_{1}\right)\right) \otimes \chi_{G_{2}}(\lambda-1)\right\} \\
= & \operatorname{det}\left\{\left(\lambda-r_{1} n_{2}-2 r_{1} \chi_{G_{2}}(\lambda-1)\right) I_{n_{1}}-\left(1-\chi_{G_{2}}(\lambda-1)\right) L\left(G_{1}\right)\right\} \\
= & f_{G_{1}}\left(\frac{\lambda-r_{1} n_{2}-2 r_{1} \chi_{G_{2}}(\lambda-1)}{1-\chi_{G_{2}}(\lambda-1)}\right)\left[1-\chi_{G_{2}}(\lambda-1)\right]^{n_{1}},
\end{aligned}
$$

where $R\left(G_{1}\right) R\left(G_{1}\right)^{T}=2 r_{1} I_{n_{1}}-L\left(G_{1}\right)$. Hence, the Laplacian characteristic polynomial of $G$ is $f_{G}(\lambda)=\left[f_{G_{2}}(\lambda-2)\right]^{m_{1}} f_{G_{1}}\left(\frac{\lambda-r_{1} n_{2}-2 r_{1} \chi_{G_{2}}(\lambda-1)}{1-\chi_{G_{2}}(\lambda-1)}\right)\left[1-\chi_{G_{2}}(\lambda-1)\right]^{n_{1}}$.

The following Theorem 4.2, first addressed in [9], is an immediate consequence of Theorem 4.1. We remark that here our method is straight-forward and different from that of Theorem 2.4 . 
Theorem 4.2. Let $G_{1}$ be an $r_{1}$ - regular graph with $n_{1}$ vertices, $m_{1}$ edges and $G_{2}$ be any graph with $n_{2}$ vertices, $m_{2}$ edges. Suppose that $L\left(G_{1}\right)=\left(\mu_{1}, \mu_{2}, \cdots, \mu_{n_{1}}\right)$, $L\left(G_{2}\right)=\left(\delta_{1}, \delta_{2}, \cdots, \delta_{n_{2}}\right)$. Then the Laplacian spectrum of $G=G_{1} \diamond G_{2}$ is given by

(i) The eigenvalue $\delta_{j}+2$ with multiplicity $m_{1}$ for every non-maximum eigenvalue $\delta_{j}\left(j=2, \ldots, n_{2}\right)$ of $L\left(G_{2}\right)$,

(ii) Two multiplicity-one eigenvalues $\frac{r_{1} n_{2}+\mu_{i}+2 \pm \sqrt{\left(r_{1} n_{2}+\mu_{i}+2\right)^{2}-4\left(n_{i}+2\right) \mu_{i}}}{2}$ for each eigenvalue $\mu_{i}\left(i=1,2, \ldots, n_{1}\right)$ of $L\left(G_{1}\right)$ and

(iii) The eigenvalue 2 with multiplicity $m_{1}-n_{1}$ (if possible).

Proof. Since the sum of all entries on every row of Laplacian matrix is zero, we have $L\left(G_{2}\right) j_{n_{2}}=0 j_{n_{2}}$, and then $\chi_{G_{2}}(\lambda)=\frac{n_{2}}{\lambda-1}$. The only pole of $\chi_{G_{2}}(\lambda)$ is $\lambda=1$, which is equivalent to the minimial Laplacian eigenvalue $\lambda-2=0$ of $G_{2}$.

Suppose that $\lambda$ is not the only pole of $\chi_{G_{2}}(\lambda)$. By theorem 4.1, one has:

(i) The $m_{1}\left(n_{2}-1\right)$ eigenvalues are $\delta_{j}+2$ with multiplicity $m_{1}$ for every non-minnimal eigenvalue $\delta_{j}\left(j=2, \ldots, n_{2}\right)$ of $L\left(G_{1}\right)$

(ii) The $2 n_{1}$ eigenvalues are obtained by solving $\lambda-r_{1} n_{2}-2 r_{1} \frac{n_{2}}{\lambda-2}=\mu_{i}\left(1-\frac{n_{2}}{\lambda-2}\right)$ for each eigenvalue $\mu_{i}\left(i=1, \ldots, n_{1}\right)$.

(iii) Now we obtain $m_{1}\left(n_{2}-1\right)+2 n_{1}$ Laplacian eigenvalues of $G$. The other $n_{1}+$ $m_{1} n_{2}-m_{1}\left(n_{2}-1\right)-2 n_{1}=m_{1}-n_{1}$ Laplacian eigenvalues of $G$ must come from the only pole $\lambda=2$ of $\chi_{G_{2}}(\lambda-1)$. This completes the proof of the theorem.

Acknowledgment: I am grateful to the anonymous referees for some friendly and helpful revising suggestions.

\section{REFERENCES}

[1] S. Barik, S. Pati, B. K. Sarma, The spectrum of the corona of two graphs, SIAM J. Discrete Math. 24 (2007), 47-56.

[2] F. Harary, Graph Theory, Addison-Wesley, Reading, PA, 1969.

[3] C. McLeman, E. McNicholas, Spectra of coronae, Linear Algebra Appl. 435 (2011), 998-1007.

[4] D. Cvetkovic, M. Doob, H. Sachs, Spectra of Graphs: Theory and Application, Johann Ambrosius Barth Verlag, Heidelberg-Leipzig, 1995.

[5] R. Merris, Laplacian matrices of graphs: a survey, Linear Algebra Appl. 197/198 (1994), $143-176$.

[6] B. Mohar, The Laplacian spectrum of graphs, Graph Theory, Combinatorics and Applications John Wiley, New York, pp. 871-898, 1991.

[7] Y. Hou, W.-C. Shiu, The spectrum of the edge corona of two graphs, Electron. J. Linear Algebra 20 (2010), 586-594.

[8] R. A. Horn and C. R. Johnson, Topics in Matrix Analysis, Cambridge University Press, Cambridge, 1991.

[9] Y. Hou, W.-C. Shiu, The spectrum of the edge corona of two graphs, Electron. J. Linear Algebra 20 (2010), 586-594.

[10] S.-L. B. Zhou, The signless Laplacian spectra of the corona and edge corona of two graphs, Linear and Multilinear Algebra 20 (2012), 1-8. 
${ }^{1}$ Department of Mathematics and Statistics, Hexi University, Zhangye, Gansu, P. R. China

E-mail address: Liuqun09@yeah.net

${ }^{2}$ QUN LIU

Department of Mathematics and Statistics,

Lanzhou University, Lanzhou, Gansu, P. R. China

E-mail address: Liuqun09@yeah.net 